\title{
Inhibition of return in patients with obsessive-compulsive disorder
}

\author{
Steffen Moritz ${ }^{\mathrm{a}, *}$, Adrian von Mühlenen ${ }^{\mathrm{b}}$ \\ ${ }^{a}$ Hospital for Psychiatry and Psychotherapy, University Hospital of Hamburg, \\ Klinik für Psychiatrie und Psychotherapie, Martinistraße 52, \\ D-20246 Hamburg, Germany \\ ${ }^{\mathrm{b}}$ Department of Psychology, University of British Columbia, 2136 West Mall, \\ Vancouver, BC, Canada V6T1Z4
}

Received 24 March 2003; received in revised form 12 November 2003; accepted 17 November 2003

\begin{abstract}
The present study is aimed at replicating and extending previous results by Nelson et al. [Psychiatry Res. 49 (1993) 183], who found decreased inhibition of return (IOR) in patients with obsessive-compulsive disorder (OCD). Thirty OCD patients, 14 psychiatric, and 14 healthy controls participated in a visual cueing experiment. The task required detection of a target stimulus at one of two possible locations. Prior to the target, an uninformative cue appeared at one of these two locations. The Stimulus Onset Asynchrony (SOA) between the cue and the target was systematically varied. We were especially interested in whether severity of OCD symptoms would be negatively correlated with inhibition for previously occupied locations. In accordance with prior research on healthy participants all groups displayed a comparable response pattern: facilitation at the short SOA condition and increasing IOR for the longer SOA conditions. Medication, comorbid depression, and OCD severity did not consistently moderate these effects.
\end{abstract}

(C) 2003 Elsevier Inc. All rights reserved.

Keywords: Obsessive-compulsive disorder; Inhibition of return; Disinhibition; Attention

\footnotetext{
* Corresponding author. Tel.: +49-40-42803-6565; fax: +49-40-42803-2999.

E-mail address: moritz@uke.uni-hamburg.de (S. Moritz).
} 


\section{Introduction}

Obsessive-compulsive disorder (OCD) is a multi-faceted psychiatric disease, which is characterized by persistent and distress-causing cognitions (obsessions) that trigger repetitive behaviors or mental acts such as washing and counting in the majority of patients (compulsions). These two psychopathological clusters are usually functionally connected and typically deal with themes of aggression, contamination, symmetry, order and religion (see Summerfeldt, Richter, Antony, \& Swinson, 1999).

Cognitive research into the pathogenesis of OCD has mainly focused on executive and inhibitory tasks with a (putative) sensitivity to the frontal lobe (see Cox, 1997; Kuelz, Hohagen, \& Voderholzer, in press; Tallis, 1997, for reviews). The interest in frontal lobe functioning stems from a number of behavioral similarities between patients with frontal damage and OCD patients (see Joseph, 1999; Lezak, 1995). Several case studies suggest that acquired brain dysfunctions in the (orbito-) frontal cortex can mimic OCD symptomatology (e.g., Eslinger \& Damasio, 1985). Interestingly, pre-existing OCD symptoms have been stopped or attenuated in some cases by frontal brain lesions (Solyom, Turnbull, \& Wilensky, 1987). Moreover, a growing number of imaging studies provide evidence for the claim that frontal cortical areas are affected in OCD, especially the anterior cingulate and the orbito-frontal region (Saxena, Brody, Schwartz, \& Baxter, 1998).

So far, the cognitive literature on executive processes in OCD has largely remained inconsistent (Cox, 1997; Tallis, 1997). In a recent study (Moritz et al., 2001a) dysfunctions in the Wisconsin Card Sorting Test and the Trail-Making Test B (set shifting) were found to be secondary to OCD and rather reflective of comorbid depressive symptoms. There is also indication that disturbances in other tasks sensitive to frontal lobe dysfunction, such as the Stroop task and verbal fluency tasks, are confined to only a subgroup of patients (Moritz et al., 2002). Convergent evidence for a strong and possibly disease-specific dysfunction, however, has been reported for performance in the Delayed Alternation Task (Abbruzzese, Bellodi, Ferri, \& Scarone, 1995; Abbruzzese, Ferri, \& Scarone, 1997; Cavedini, Ferri, Scarone, \& Belodi, 1998; Moritz, Fricke, \& Hand, 2001b), which is thought to tap into orbito-frontal functioning. There is also some indication that performance in a number of spatial tasks (mental rotation, visuospatial transformation) is decreased in OCD, although the specificity of dysfunction in this domain awaits further establishment (Moritz, Kloss, Jahn, Schick, \& Hand, 2003; see Kuelz et al., in press, for a review).

No conclusive results have emerged for tasks tapping into inhibitory control. ${ }^{1}$ Prominent questions for this line of research include whether (a) the emergence of

\footnotetext{
${ }^{1}$ It may appear artificial to distinguish between executive/"frontal lobe" tasks and tests assessing inhibitory control. While the former tests reflect a more anatomy-based tradition in neurocognitive research, the latter line of research is more concerned with underlying, functionally meaningful processes than with cortical substrates.
} 
OCD symptoms reflects an inability to inhibit (irrelevant) cognitions that would normally not enter consciousness, and/or whether (b) mental and motor acts in OCD have a longer resting time as a consequence of greater initial activation or reduced late-onset inhibition. The former assumption has been addressed using the negative priming (NP) paradigm (Tipper, 1985). While Enright and Beech (1990, 1993a, 1993b) found a significant reduction of NP in OCD patients suggestive of impaired cognitive inhibition, these results have not been replicated subsequently (e.g., MacDonald, Antony, MacLeod, \& Swinson, 1999).

Another phenomenon relevant to the study of inhibitory processes in OCD patients is inhibition of return (IOR). IOR was first demonstrated by Posner and Cohen (1984) with a spatial cueing paradigm. They found that it takes longer to redirect attention to a previously attended location, than to an unattended location. Whether this effect is based on visual or motor processes has been subject to a large debate in the course of the last two decades (for an overview see Taylor \& Klein, 2000). Nonetheless the effect itself has turned out to be fairly robust (see Klein, 2000, for a recent review), and it has been replicated across different modalities (e.g., Spence \& Driver, 1998) and paradigms (e.g., Klein, 1988; Müller $\&$ von Mühlenen, 2000). All of these findings support the view that IOR taps into the disengagement of attention to a particular location in order to facilitate the redirection of the cognitive system to other novel locations.

We only know of one study that has investigated IOR in OCD patients. Nelson, Early, and Haller (1993) compared 15 OCD patients with 15 healthy controls and found a marked reduction in IOR in the patient group. While IOR was decreased for left visual field targets, reversed IOR (i.e., facilitation) was observed for right visual field targets in OCD patients, which according to Nelson et al. may point to a lateralized brain stem lesion or a malfunctioning of the retinotectal pathway in OCD.

The present study attempted to confirm and extend the initial findings collected by Nelson et al. (1993). One shortcoming in Nelson et al.'s study was the lack of a psychiatric control group. In order to examine specificity of a putative inhibition deficit in IOR we introduced a psychiatric control group consisting of patients with anxiety disorders other than OCD. Nelson et al. also concede that the impact of depressive and OCD symptoms were not thoroughly after studied. Therefore, we looked at these possible moderators, along with medication and other potentially confounding variables. Finally, by using three intervals instead of two, as in the study of Nelson et al., the present experiment allowed for a more fine-graded estimation of the time course of IOR in OCD patients and controls.

\section{Methods}

\subsection{Participants}

Thirty patients fulfilling a diagnosis of OCD participated in the study (10 males, 20 females; age: 31.83 (S.D.: 9.67); years of education: 11.53 (S.D.: 2.08); 
number of previous hospitalizations: 1.23 (S.D.: 1.53); length of illness: 7.79 years (S.D.: 7.47); Hamilton Depression Rating Scale (HDRS, 17 items): 6.07 (S.D.: 4.37); Y-BOCS total score: 16.89 (S.D.: 8.21); premorbid intelligence quotient (IQ; Lehrl, 1995): 110.20 (S.D.: 15.98); medicated: 17 patients). The healthy control group consisted of 14 participants (4 males, 10 females; age: 27.14 (S.D.: 5.08); years of education: 12.43 (S.D.: 1.70); premorbid IQ: 111.29 (S.D.: 12.36)). The 14 psychiatric controls were suffering from an anxiety disorder other than OCD. Most of the patients in this group were either treated for panic disorder $(n=10)$ or post-traumatic stress disorder (PTSD) $(n=4)$ (4 males, 10 females; age: 33.71 (S.D.: 7.68); years of education: 11.14 (S.D.: 1.75); number of previous hospitalizations: 0.64 (S.D.: 0.84) length of illness: 5.21 years (S.D.: 4.02); HDRS: 8.69 (S.D.: 5.97); premorbid IQ: 108.00 (S.D.: 9.69); medicated: 3 patients). Participants did not differ significantly on any socio-demographic background variable or premorbid intelligence (all Bonferroni-corrected comparisons at least $P>.1$ ).

Psychiatric diagnoses were determined according to DSM-IV criteria using a semi-structured interview: (Mini International Neuropsychiatric Interview: Lecrubier et al., 1997). In addition to this, medical records were carefully screened for symptoms incompatible with a diagnosis of OCD. Participants did not reveal a history of comorbid drug abuse, substantial neurological disorder (e.g., stroke, multiple sclerosis, head trauma, previous brain operations), current or previous psychotic symptoms. Obsessive-compulsive symptoms were assessed with the Yale-Brown Obsessive-Compulsive Scale (Y-BOCS; Goodman et al., 1989; German translation by Hand \& Büttner-Westphal, 1991). Scores for obsessions and compulsions were computed using a recently proposed algorithm (obsessions: items 1-3; compulsions: 6-8; resistance against symptoms: 4 and 9; Moritz et al., 2002).

Healthy controls were drawn from hospital staff and the general population. Healthy participants were screened for psychopathological disturbances or substance abuse. All participants gave written informed consent to participate after they had been fully informed about the study.

\subsection{Apparatus}

Stimuli were presented on a Sony 17 -in. Trinitron monitor with a graphic resolution of $640 \times 480$ pixel, driven by a Pentium II $300 \mathrm{MHz}$ PC. The room was dimly illuminated to prevent reflections on the monitor. Participants viewed the monitor from a distance of approximately $60 \mathrm{~cm}$. Responses were recorded using the right button of a serial Microsoft mouse.

\subsection{Display and stimuli}

The display contained three square boxes (size $1.6^{\circ}$ visual angle), horizontally arranged (distance between boxes $8.8^{\circ}$ visual angle). Box lines where drawn in 


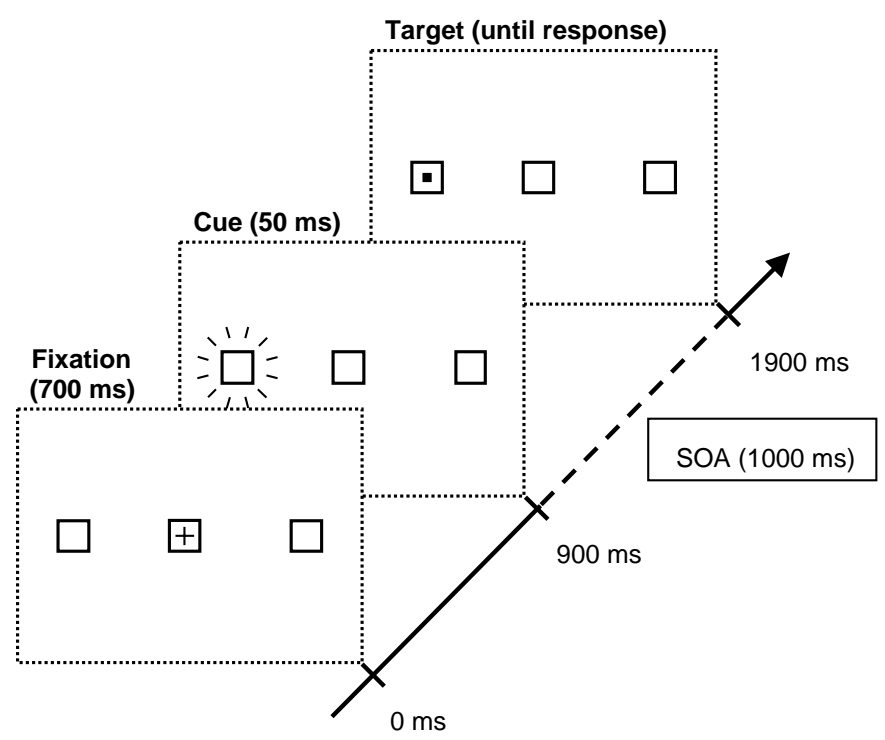

Fig. 1. Sequence of events in a valid trial showing the cue and the target in the left peripheral box. The arrow line represents the time axis. The broken axis line stands for varying SOAs, which was $1000 \mathrm{~ms}$ in this example.

dark gray on a black background with a thickness of 1 pixel. The fixation cross (size $0.8^{\circ}$ visual angle) was presented in the middle of the center box. The cue-a short flashing of one of the peripheral boxes-was created by changing the color of the box from dark gray to white (for $50 \mathrm{~ms}$ ). The target, a small light gray filled square (size $0.25^{\circ}$ visual angle), was shown in the center of one of the two peripheral boxes (see Fig. 1).

\subsection{Procedure and design}

The sequence of events in a trial is shown in Fig. 1. The three boxes were shown permanently throughout a block of trials. A trial started with presentation of a central fixation cross for $700 \mathrm{~ms}$, followed by a $200 \mathrm{~ms}$ blank period with only the three boxes. The cue was presented for $50 \mathrm{~ms}$ with equal probability at one of the two peripheral boxes. The cue was followed by the target, which also appeared equally often and independent of the cue (i.e., the cue had no predictive value for the target location) at one of the two peripheral boxes. The Stimulus Onset Asynchrony (SOA) between cue and target was systematically varied from 100, 500 to $1000 \mathrm{~ms}$. Participants were instructed to press the button as quickly as possible whenever they saw the target. The target was on the screen until the button was pressed. In $14 \%$ of the trials no target was presented and participants were instructed to refrain from pressing the button. In these catch trials the three empty boxes were shown for $1500 \mathrm{~ms}$ before the next trial started. Acoustic error 
feedback was provided in target trials when the button was pressed too early (before target onset) too late (more than $2000 \mathrm{~ms}$ after target onset), or when the button was pressed in catch trials. The inter-trial interval was $1000 \mathrm{~ms}$.

Each participant completed a total of 168 trials-144 experimental and 24 catch trials. All trials were presented in fully randomized order. The experiment was divided into 4 blocks of 42 trials, with 15 -s breaks between blocks. Each participant received a practice block consisting of 42 trials. Each block started with five unrecorded "warm-up" trials. The dependent variables were reaction time (RT) and percentage of errors. RTs smaller than $100 \mathrm{~ms}$ or larger than $1500 \mathrm{~ms}$ were counted as errors. Mean RTs were calculated individually for each participant and for each combination of target location, validity, and SOA.

\section{Results}

\subsection{Reaction time}

Mean RTs were calculated, excluding trials with RTs below or above two standard deviations from the mean (OCD: $4.7 \%$, anxiety: $4.4 \%$, healthy: $4.9 \%$ of all trials). A four-way mixed ANOVA $(3 \times 2 \times 2 \times 3)$ was conducted for RT with group (OCD, anxiety, healthy) as the between-subject variable and target location (left, right), validity (invalid, valid) and SOA $(100,500,1000 \mathrm{~ms})$ as withinsubject variables. The main effect of group did not reach significance, $F(2,110)=1.08, \mathrm{~ns}$, reflecting comparable overall reaction times across groups. The main effect of SOA was significant, $F(2,110)=3.73, P<.05$, representing overall slower responses in the $100 \mathrm{~ms}$ conditions relative to the 500 and $1000 \mathrm{~ms}$ conditions.

In order to facilitate interpretation of the various interactions, we calculated the cueing effects for each group $\times$ target location $\times$ SOA combination by subtracting the valid RTs from the invalid RTs (see Fig. 2). A positive value reflects a facilitating effect of the cue on the target, a negative value reflects an inhibitory effect of the cue on the target. Of the two-way interactions, the expected validity $\times$ SOA interaction achieved significance, $F(2,110)=51.86, P<.001$. The overall cueing effect (invalid RTs minus valid RTs) was positive (i.e., facilitation) for the $100 \mathrm{~ms}$ SOA condition, and negative (i.e., inhibition) for the 500 and $1000 \mathrm{~ms}$ SOA condition. Further, the interaction of target location $\times$ validity was significant, $F(1,55)=$ $5.58, P<.05$, which can be attributed to an overall smaller cueing effect at the left location $(-8 \mathrm{~ms})$ than at the right location $(2 \mathrm{~ms})$.

The predicted three-way group $\times$ validity $\times$ SOA interaction did not reach significance, $F(4,110)=0.36, P>.8$, suggesting that the typical cueing pattern with early facilitation followed by later inhibition effects did not differ between the three groups. However, the four-way interaction achieved significance, $F(4,110)=3.61 ; P<.05$. Planned contrasts revealed that this interaction is attributable to greater IOR for right targets relative to left targets in the anxiety 
healthy

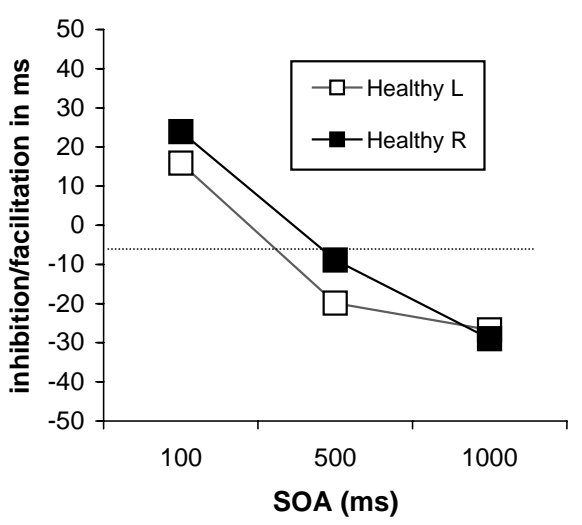

anxiety

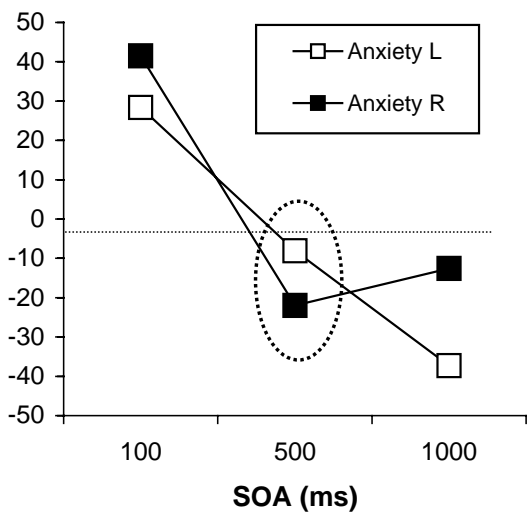

OCD

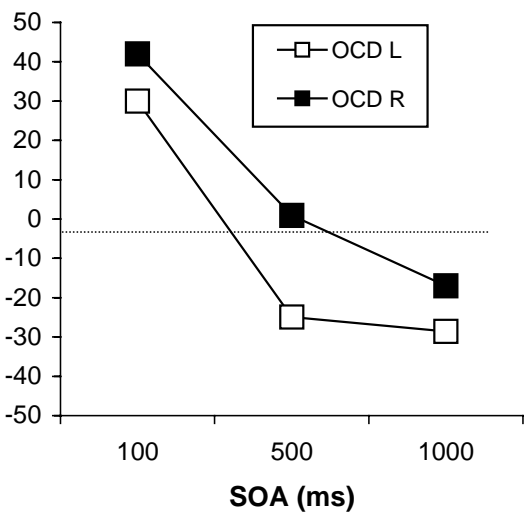

Fig. 2. All groups displayed significant facilitation at the $100 \mathrm{~ms}$ interval (faster responses for valid targets relative to invalid targets), while inhibition of return (IOR) emerged for the longer SOAs (especially for the $1000 \mathrm{~ms}$ condition). The significant four-way interaction is attributable to greater IOR for right visual targets relative to left visual targets in anxiety patients, while the opposite pattern emerged for both other groups (see ellipse). 
group at the $500 \mathrm{~ms}$ SOA condition (see marking with broken line in Fig. 2), whereas the opposite effect (i.e., smaller IOR for right vs. left targets) emerged in the OCD and healthy group (see Fig. 2).

Simple paired $t$-tests revealed that all groups displayed significant facilitation at $100 \mathrm{~ms} \mathrm{SOA} \mathrm{(all} P$ 's $<.01$ ) and significant IOR at $1000 \mathrm{~ms} \mathrm{SOA}$ (all $P$ 's $<.01$ ). At the $500 \mathrm{~ms}$ interval both patient groups failed to exhibit significant IOR (anxiety: $t(13)=1.61, P=.13$; OCD: $t(29)=1.63, P=.11)$, while controls showed significant inhibition $(t(13)=2.71, P<.05)$.

\subsection{Errors}

The overall error rate was very low $(<1 \%)$, for both misses and false alarms. A four-way mixed ANOVA model $(3 \times 2 \times 2 \times 3)$ on the misses with the factors group, target location, validity, and SOA, revealed only a significant validity $\times$ SOA interaction effect, $F(2,110)=5.36 ; P<.01$. This interaction was due to an increase in misses with SOA, which was somewhat more pronounced in invalid trials than in valid trials. No other effect reached significance.

\subsection{Correlational analyses}

We correlated IOR effects for all SOAs (both combined and separate for left and right targets) with Y-BOCS scores, HDRS total score, medication, number of hospitalizations and length of illness. Except for compulsions, which were negatively correlated with facilitation at the $100 \mathrm{~ms}$ for right visual targets $(r=-.42 ; P=.03)$, none of the correlations achieved significance. This latter correlation, however, did not withstand a Bonferroni-correction.

\section{Discussion}

The present study failed to replicate findings collected by Nelson et al. (1993). All three groups displayed a comparable time course pattern of IOR: facilitation at short intervals and increasing inhibition at longer intervals. Due to larger standard deviations both psychiatric groups failed to reach a significant inhibition effect for the $500 \mathrm{~ms}$ condition, despite a comparable mean IOR effect relative to controls. Group comparisons did not yield a significant difference. As we recruited a large OCD sample $(n=30)$, lack of statistical power cannot account for this finding. Except for a weak relationship between compulsions and increased facilitation for right visual targets which did not withstand a Bonferroni-correction, neither psychopathological nor background variables were correlated with any of the experimental parameters. We initially expected a correlation between severity of OCD symptoms and IOR, as repetitive behaviors and thoughts might stem from a disinhibition of previous mental acts or behaviors (i.e., disinhibition might lead to an echo of prior activation, while preserved inhibition would block the 
re-occurrence of a cognition or behavior after some time). However, this response pattern did not emerge.

Although our results speak against a deficit of IOR in OCD, this clearly does not justify the conclusion that inhibitory processes are intact in this disorder. "Inhibition" is an umbrella term comprising a class of different processes rather than a single cognitive mechanism. It can also be objected that IOR is a spatial task and therefore may have been less relevant for obsessions/repetitive thoughts. OCD symptoms mostly refer to recurrent scenes or thoughts rather than previously occupied localizations. For this reason, we are currently investigating whether patients with OCD have an excitation or less attenuation for activated verbal material across time. In a pilot study using a repetition priming experiment we tested the hypothesis that controls show a quicker decay of verbal activation than OCD patients. In line with this assumption, it was found that, compared to controls OCD patients exhibited a stronger repetition priming for long but not short intervals. When negative, distressing thoughts have a longer maintenance time in consciousness this may increase anxiety and the feeling of endangerment and may drive participants to seek for additional (dysfunctional) activities (e.g., compulsions) to control/reduce such cognitions.

Another fruitful approach might be to employ concern-related material for neurocognitive tasks, that is, to depart from "neutral" tasks to see how patients process affectively charged contents.

\section{References}

Abbruzzese, M., Bellodi, L., Ferri, S., \& Scarone, S. (1995). Frontal lobe dysfunction in schizophrenia and obsessive-compulsive disorder: a neuropsychological study. Brain and Cognition, 27, 202-212.

Abbruzzese, M., Ferri, S., \& Scarone, S. (1997). The selective breakdown of frontal functions in patients with obsessive-compulsive disorder and in patients with schizophrenia: a double dissociation experimental finding. Neuropsychologia, 35, 907-912.

Cavedini, P., Ferri, S., Scarone, S., \& Belodi, L. (1998). Frontal lobe dysfunction in obsessivecompulsive disorder and major depression: a clinical-neuropsychological study. Psychiatry Research, 78, 21-28.

Cox, C. (1997). Neuropsychological abnormalities in obsessive-compulsive disorder and their assessments. International Review of Psychiatry, 9, 45-59.

Enright, S. J., \& Beech, A. R. (1990). Obsessional states: anxiety disorders or schizotypes? An information processing and personality assessment. Psychological Medicine, 20, 621-627.

Enright, S. J., \& Beech, A. R. (1993a). Reduced cognitive inhibition in obsessive-compulsive disorder. British Journal of Clinical Psychology, 32, 67-74.

Enright, S. J., \& Beech, A. R. (1993b). Further evidence of reduced cognitive inhibition in obsessivecompulsive disorder. Personality and Individual Differences, 14, 387-395.

Eslinger, P. J., \& Damasio, A. R. (1985). Severe disturbance of higher cognition after bilateral frontal lobe ablation: patient EVR. Neurology, 35, 1731-1741.

Goodman, W., Lawrence, H., Price, L. H., Rasmussen, S. A., Mazure, C., Fleischmann, R. L. et al. (1989). The Yale-Brown Obsessive Compulsive Scale. I. Development, use, and reliability. Archives of General Psychiatry, 46, 1006-1011. 
Hand, I., \& Büttner-Westphal, H. (1991). Die Yale-Brown Obsessive-Compulsive Scale (Y-BOCS): ein halbstrukturiertes Interview zur Beurteilung des Schweregrades von Denk- und Handlungszwängen. Verhaltenstherapie, 1, 223-225.

Joseph, R. (1999). Frontal lobe psychopathology: mania, depression, confabulation, catatonia, perseveration, obsessive compulsions, and schizophrenia. Psychiatry, 62, 138-172.

Klein, R. (1988). Inhibitory tagging system facilitates visual search. Nature, 334, 430-431.

Klein, R. M. (2000). Inhibition of return. Trends in Cognitive Sciences, 4, 138-147.

Kuelz, A. K., Hohagen, F., \& Voderholzer, U. (in press). Neuropsychological dysfunction in obsessive-compulsive disorder: a critical review. Biological Psychology.

Lecrubier, Y., Sheehan, D., Weiller, E., Amorim, P., Bonora, I., Sheehan, K. et al. (1997). The MINI International Neuropsychiatric Interview (MINI), a short diagnostic interview: reliability and validity according to the CIDI. European Psychiatry, 12, 224-231.

Lehrl, S. (1995). Mehrfachwahl-Wortschatz-Intelligenztest: MWT-B (3. Auflage ed.). PERIMEDspitta: Balingen.

Lezak, M. D. (1995). Neuropsychological assessment (3rd ed.). Oxford: Oxford University Press.

MacDonald, P. A., Antony, M. M., MacLeod, C. M., \& Swinson, R. P. (1999). Negative priming for obsessive-compulsive checkers and noncheckers. Journal of Abnormal Psychology, 108, 679-686.

Moritz, S., Birkner, C., Kloss, M., Fricke, S., Böthern, A., \& Hand, I. (2001a). Impact of comorbid depressive symptoms on neuropsychological performance in obsessive-compulsive disorder. Journal of Abnormal Psychology, 110, 653-657.

Moritz, S., Fricke, S., \& Hand, I. (2001b). Further evidence for delayed alternation deficits in obsessive-compulsive disorder. Journal of Nervous and Mental Disease, 189, 562-564.

Moritz, S., Kloss, M., Jacobsen, D., Wein, C., Fricke, S., \& Hand, I. (2002). Dimensional structure of the Yale-Brown Obsessive Compulsive Scale (Y-BOCS). Psychiatry Research, 109, 193-199.

Moritz, S., Kloss, M., Jahn, H., Hand, I., Haasen, C., \& Krausz, M. (2002). Executive functioning in obsessive-compulsive disorder, unipolar depression and schizophrenia. Archives of Clinical Neuropsychology, 17, 477-483.

Moritz, S., Kloss, M., Jahn, H., Schick, M., \& Hand, I. (2003). Impact of comorbid depressive symptoms on nonverbal memory and visuospatial performance in obsessive-compulsive disorder. Cognitive Neuropsychiatry, 8, 261-272.

Müller, H.J., \& von Mühlenen, A. (2000). Probing distractor inhibition in visual search: inhibition of return. Journal of Experimental Psychology: Human Perception \& Performance, 26, 1591-1605.

Nelson, E., Early, T. S., \& Haller, J. W. (1993). Visual attention in obsessive-compulsive disorder. Psychiatry Research, 49, 183-196.

Posner, M. I., \& Cohen, Y. (1984). Components of visual orienting. In: H. Bouma \& D. Bouwhuis (Eds.), Attention and performance (Vol. X, pp. 531-556). Erlbaum: London.

Saxena, S., Brody, A. L., Schwartz, J. M., \& Baxter, L. R. (1998). Neuroimaging and frontal-subcortical circuitry in obsessive-compulsive disorder. British Journal of Psychiatry, 173(Suppl. 35), 26-37.

Solyom, L., Turnbull, I. M., \& Wilensky, M. (1987). A case of self-inflicted leucotomy. British Journal of Psychiatry, 151, 855-857.

Spence, C., \& Driver, J. (1998). Auditory and audiovisual inhibition of return. Perception \& Psychophysics, 60, 125-139.

Summerfeldt, L. J., Richter, M. A., Antony, M. M., \& Swinson, R. P. (1999). Symptom structure in obsessive-compulsive disorder: a confirmatory factor-analytic study. Behaviour Research and Therapy, 37, 297-311.

Tallis, F. (1997). The neuropsychology of obsessive-compulsive disorder: a review and consideration of clinical implications. British Journal of Clinical Psychology, 36, 3-20.

Taylor, T. L., \& Klein, R. M. (2000). Visual and motor effects in inhibition of return. Journal of Experimental Psychology: Human Perception and Performance, 26, 1639-1656.

Tipper, S. P. (1985). The negative priming effect: Inhibitory priming by ignored objects. The Quarterly Journal of Experimental Psychology, 37A, 571-590. 